\title{
Improving physical activity after stroke via treadmill training and self management (IMPACT): a protocol for a randomised controlled trial
}

\author{
Sandra G. Brauer ${ }^{1 *}$, Suzanne S. Kuys ${ }^{2}$, Jennifer D. Paratz ${ }^{3}$ and Louise Ada ${ }^{4}$
}

\begin{abstract}
Background: The level of physical activity undertaken by stroke survivors living in the community is generally low. The main objectives of the IMPACT trial are to determine, in individuals undergoing rehabilitation after stroke, if 8 weeks of high-intensity treadmill training embedded in self-management education (i) results in more physical activity than usual physiotherapy gait training and (ii) is more effective at increasing walking ability, cardiorespiratory fitness, self-efficacy, perception of physical activity, participation, and health-related quality of life as well as decreasing cardiovascular risk, and depression, at 8 and 26 weeks.
\end{abstract}

Methods: A prospective, two-arm, parallel-group, randomised trial with concealed allocation, blinded measurement and intention-to-treat analysis, will be conducted. 128 stroke survivors undergoing rehabilitation who are able to walk independently will be recruited and randomly allocated to either the experimental or control group, who will both undergo gait training for 30 min, three times a week for 8 weeks under the supervision of a physiotherapist. Outcomes will be measured at baseline (Week 0), on completion of the intervention (Week 8) and beyond the intervention (Week 26). This study has obtained ethical approval from the relevant Human Research Ethics Committees.

Discussion: Improving stroke survivors' walking ability and cardiorespiratory fitness is likely to increase their levels of physical activity. Furthermore, if education in self-management results in sustained high levels of physical activity, this should result in improved participation and quality of life.

Trial registration: This trial was registered with the Australian New Zealand Clinical Trials Registry (ACTRN12613000744752) on 4th July, 2013.

Keywords: Stroke, Randomised, Treadmill, Walking, Cardiorespiratory fitness, Physical activity

\section{Background}

Stroke survivors have three times the risk of further cardiovascular events than the general population $[1,2]$. There is strong evidence that physical activity has a protective effect against stroke [3, 4], it can reduce cardiovascular risk factors in people with stroke [5] and not undertaking enough physical activity increases the risk of recurrent stroke [6, 7]. The level of physical activity undertaken by stroke survivors living in the community is generally low $[8,9]$; less than

\footnotetext{
* Correspondence: s.brauer@uq.edu.au

'Discipline of Physiotherapy, School of Health and Rehabilitation Sciences,

The University of Queensland, St Lucia, QLD, Australia

Full list of author information is available at the end of the article
}

half that of age-matched adults $[10,11]$. However, increasing the level of physical activity after stroke is challenging, perhaps because multiple factors contribute. For example, lack of physical activity is correlated with poor cardiorespiratory fitness [12], reduced walking ability [13] and low self-efficacy [14].

High-intensity treadmill training, i.e., training to a target heart rate around $40-60 \%$ of heart rate reserve, has been shown to increase cardiorespiratory fitness in people with chronic stroke $[15,16]$. However, this type of training is not usually started during inpatient rehabilitation. In a pilot study, we have shown that high-intensity treadmill training during inpatient rehabilitation is feasible, is not 
detrimental to the walking pattern and may result in an improvement in walking ability [17]. However, we have not shown that it increases cardiorespiratory fitness.

Furthermore, one of the main findings of studies investigating interventions to improve cardiorespiratory fitness after stroke, is that the benefit is only partially maintained beyond the period of intervention [15]. It is therefore important to support stroke survivors to exercise independently in the long term [18]. A self-management approach to rehabilitation has been recommended as a strategy to optimise outcomes for stroke survivors after discharge from hospital [19]. Interventions that are grounded in health behaviour theory, and which target psychological constructs with evidence based intervention activities, are more successful than those which are neither theory- nor evidence-based [20]. The Health Action Process Approach (HAPA) has been found to be useful, particularly in the rehabilitation setting, for predicting and explaining changes in health behavior [20]. It consists of two phases - a motivational phase and a volitional phase. In the motivational phase, the perception that a person is at risk, their positive outcome expectancies for completing a certain behaviour, and their perceived self-efficacy for performing a certain action interact to drive the formation of a behaviour intention [20]. In the volitional phase, selfregulatory skills and strategies (action planning, coping planning, recovery self-efficacy and social support) are employed to translate intentions into action [20]. Intervention behaviour change techniques can be tailored to participants dependent on their phase. The validity of the HAPA has been tested in the intervention context with people in the rehabilitation setting, including individuals with stroke [21,22] and could be useful to address the behavior change issue of modifying physical activity after stroke. To augment this approach, goal setting theory can be employed to target goal difficulty and specificity [23].

It appears that a comprehensive, patient-centered, goaloriented approach to lifestyle modification is required early in rehabilitation that addresses both the ability for stroke survivors to be active, and the motivation to do so. Maintaining high levels of physical activity via improved walking ability and ongoing cardiorespiratory fitness should also confer a range of benefits, such as an improvement in self-efficacy, participation, and health-related quality of life and a decrease in cardiovascular risk [24], and depression. If a sufficient training level can be reached, there is likely to be an impact on lipid profile: high sensitivity C- reactive protein (HSCRP), total cholesterol (TC), triglycerides (TRG) high density and lipoprotein cholesterol (HDL-C) and low density lipoprotein cholesterol (LDL-C). The link between modification of the lipid profile and inflammatory biomarkers to carotid intima-media thickness and subsequent cerebro and cardiovascular events has been convincingly demonstrated
[25]. Additionally, there is strong evidence that moderate intensity exercise can improve lipid profiles and inflammatory markers, including highly sensitive $\mathrm{C}$-reactive protein (HSCRP), an independent predictor of cardiovascular events in both primary and secondary prevention [26-29]. However, until recently it has been difficult to exercise stroke patients (who due to their pathophysiology are already at risk of future cardiovascular events), at a sufficient level to achieve these benefits. A recent study [30] has provided the first evidence that high-intensity treadmill training in chronic stroke survivors reduces cardiovascular risk markers (cerebral vasomotor reactivity), implying that this form of exercise intervention may confer a neuroprotective effect. High-intensity treadmill training may lower cardiovascular disease risk factors for stroke survivors which in turn should result in secondary prevention of future cardiovascular and cerebrovascular events.

We are therefore conducting a randomized trial investigating the effect of high-intensity treadmill training during inpatient rehabilitation embedded in self-management. This will be compared with usual physiotherapy gait training to ensure a similar volume of training. The main objectives of the IMPACT trial are to determine, in individuals undergoing rehabilitation after stroke, if 8 weeks of highintensity treadmill training embedded in self-management:

(i) results in more physical activity than usual gait training; and

(ii) is more effective at increasing walking ability, cardiorespiratory fitness, self-efficacy, perception of physical activity, participation, and quality of life as well as decreasing cardiovascular risk, and depression; immediately after the intervention at 8 weeks and beyond the intervention at 6 months.

\section{Methods}

A prospective, two-arm, parallel-group, randomised trial with concealed allocation, blinded measurement and intention-to-treat analysis will be undertaken with stroke survivors undergoing rehabilitation (Fig. 1). Participants will be recruited from rehabilitation units in two states (Queensland and NSW) in Australia. A list of centres is available on the trial registry. Participants will be randomly allocated to two groups. Gait training for the experimental group will be high-intensity treadmill training plus self management and for the control group will be usual gait training. Both groups will have the same amount of time spent on gait training. To minimise the risk of contamination, the experimental group will receive their intervention from a different therapist in a different location from the control group. The rest of the multidisciplinary rehabilitation program will be unchanged. The end point of the intervention phase of the study will be 8 weeks and the end point of the follow-up phase will 


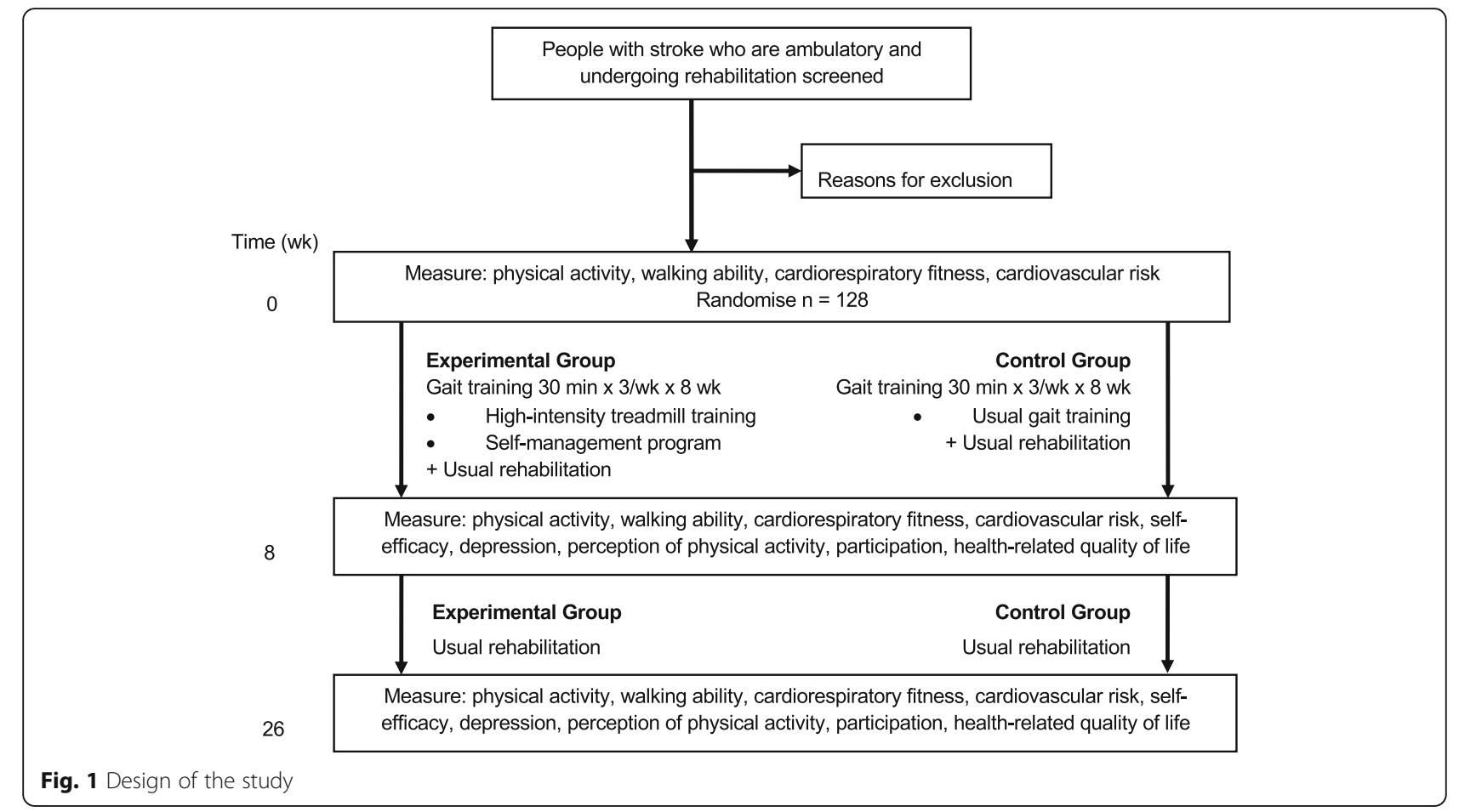

be 26 weeks. Outcomes will be measured at baseline (Week 0), on completion of the intervention (Week 8) and beyond the intervention (Week 26). Both data collection and data analysis will be completed by researchers who are blind to group allocation. Participants will be asked not to reveal the nature of their intervention to measurers. It is not possible to blind participants or therapists to group allocation. The study protocol has been approved by the appropriate Human Research Ethics Committees and has been registered on the Australian New Zealand Clinical Trials Registry.

\section{Participants, therapists, centres}

Individuals with stroke will be screened and invited to participate if they are: within two months of a stroke (confirmed by CT scan or clinical diagnosis); aged 18 years or older; able to walk $10 \mathrm{~m}$ independently (without physical assistance but with or without an aid); and able to follow three-stage commands. They will be excluded if they: were unable to walk independently prior to current stroke; have co-morbidities relating to the lower limbs that might limit their walking (such as arthritis); have unstable cardiac status (which would preclude them from participating in intensive training); unable to return for assessment or training; or are unable to provide informed consent. Information that will be collected to describe the sample will include: age, sex, description of the stroke (time, side affected, type, location, severity), aphasia, recombinant tissue plasminogen activator (rt-PA), medications, co-morbidities, and previous living arrangements.
Therapists delivering the intervention will be included if they are physiotherapists with more than 5 years clinical experience, or experience in treadmill training or self-management.

Centres will be included if they contain a rehabilitation unit that has a throughput of stroke of more than 50 per year.

\section{Randomisation}

Randomisation will be computer-generated, independent and concealed. The allocation sequence has been computergenerated by an independent researcher not involved in this study. For each centre, allocation to the experimental or control group will occur in random permuted blocks, so that after every block (of 4-6 participants), the experimental and control group will contain equal numbers [31]. Group allocation is stored in numbered, opaque envelopes in order to provide secure randomisation. After baseline measures have been collected, the therapist providing the intervention opens the envelope and reveals the group allocation. In this way, the allocation sequence is concealed from on-site recruiters.

\section{Intervention}

Both the experimental and the control group will undergo gait training during rehabilitation as part of this trial for $30 \mathrm{~min}$, three times a week for 8 weeks under the supervision of a therapist. Gait training for the other part of the week will be as usual. The gait training may be carried out across inpatient and outpatient departments - since some 
participants may be discharged home before the end of the 8-week training. No other part of the multidisciplinary rehabilitation program will be controlled. Randomisation should ensure that any effect of other interventions will be the same for both groups, therefore, other rehabilitation will not be withheld. Typically for stroke patients, rehabilitation broadly consists of as much physical practice as possible including early mobilisation and upper limb training as specified by the Clinical Guidelines for Stroke Management 2010 [32].

\section{Experimental group}

The experimental group will receive high-intensity treadmill training and self-management education. The highintensity treadmill training will be based on our pilot program [17] of up to 30 min walking on a treadmill at an intensity of $40-60 \%$ heart rate reserve or a Borg Rating of Perceived Exertion [33] of 11-14; the minimum required for training cardiorespiratory fitness [34]. Target heart rates will be calculated according to Karvonen method [34] adjusting for beta-blockers for those taking heart rate lowering medication. Participants will start at an intensity of $40 \%$ heart rate reserve, progressing each week by a $5-10 \%$ increase until $60 \%$ heart rate reserve is reached. Heart rate will be recorded via a portable heart rate monitor. While on the treadmill, participants will be encouraged to hold the handrail and a physiotherapist will provide assistance as required to ensure foot clearance during swing phase. In addition, an assistant will be available to operate the controls and the safety stop cord will be attached to participants at all times.

The self-management education in this intervention is underpinned by two theoretical models: the Health Action Process Approach (HAPA) [35] and Goal Setting Theory [36]. At the beginning of the study, participants in the treadmill training group will receive a detailed workbook to complement the treadmill training component of the intervention. The workbook will serve a dual purpose of encouraging self-monitoring of physical activity and guiding participants through the process of setting short- and long-term goals as well as formulating action plans and coping strategies. The therapist delivering the intervention will allocate a proportion of each treadmill session to addressing the self-management-related tasks outlined in the workbook. The multi-modal behaviour change techniques to be employed have been selected to align with the theoretical basis of the intervention and progress over time. These include: education, behavioural instruction, self-monitoring, goal setting and goal review, feedback, problem solving, action planning and coping planning. The way in which these behaviour changes strategies are operationalized is described below. In order to address risk perception and outcome expectancies, participants will be informed about the consequences of their action (inaction) and asked to consider the costs and benefits of their behaviour. Task self-efficacy (for treadmill walking and physical activity generally) will be enhanced by providing instruction on how to perform the behaviour, prompting of self-monitoring (using the workbook), and the provision of feedback on performance. Participants will be asked to set specific, relevant and patient-centred short- medium- and long-term goals as a way of fostering intention formation. Action planning and coping planning (i.e., problem solving and identifying strategies to overcoming potential barriers to achieving behaviour change goals) will be used to build self-efficacy for behaviour maintenance. As participants reach the end of the treadmill intervention, and transition to independent physical activity practice, further strategies to encourage maintenance selfefficacy will be employed. These will include discussions to prompt participants to review their behavioural goals and gather information on alternative options to incorporate sufficient physical activity program in their life.

Physiotherapists delivering the experimental intervention will be trained in high-intensity treadmill training and self-management education and provided with guidelines, training manuals and support. Information describing the high-intensity treadmill training (e.g., duration, distance walked, observed heart rate, perceived exertion, adverse events) and self-management education (e.g. frequency of participant-initiated conversations on coping planning) will be recorded to be able to describe the intervention accurately. Adherence to the experimental intervention will be monitored by reviewing the recording sheets and by auditing $1-2$ sessions per therapist.

\section{Control group}

The control group will receive usual gait training delivered by the usual treating physiotherapist. Usual gait training will not be prescribed, but quantified (e.g., time and distance walked, tasks practiced will be recorded) to be able to describe the intervention accurately. The recordings of usual gait training at the beginning and the end of the study end will be examined to check for contamination from the experimental intervention.

\section{Measurement}

Since the main aim the study is to improve physical activity, the primary outcome will be physical activity measured as steps/day over four days at Weeks $0,8,26$. Secondary measures collected at Weeks 0,8 and 26 will include walking ability, cardiorespiratory fitness and cardiovascular risk. Secondary measures collected at Weeks 8 and 26 will include self-efficacy, depression, perception of physical activity, participation, and health-related quality of life. 


\section{Primary}

- Physical activity will be measured over 4 days using a triaxial accelerometer (activPAL3 ${ }^{\mathrm{Tm}}$, PAL Technologies Ltd., Glasgow, UK) secured to the middle of the anterior non-paretic thigh and reported as steps/day. This device has been shown to demonstrate concurrent validity for step count, by demonstrating low error (1.6\%) and high concurrence with direction observation (ICC 0.99, 95\% CI 0.98 to 1.0) in people with stroke walking in an outdoor circuit [37], and test-retest reliability when walking indoors across days (ICC 0.98, 95\% CI 0.93-0.99) [37].

\section{Secondary}

- Walking ability will be measured using the 6-min Walk Test and the 10-m Walk Test. The 6-min Walk Test will be conducted according to the American Thoracic Society guidelines [38] and reported as distance walked in $\mathrm{m}$. It is commonly used as a functional submaximal measure of oxygen uptake and exercise capacity [39] and is a valid and reliable clinical measure of walking ability in stroke survivors [40]. The 10-m Walk Test will be conducted at preferred and fast speeds over a $15 \mathrm{~m}$ course to remove any acceleration and deceleration, using usual gait aids, and reported as mean (SD) speed in $\mathrm{m} / \mathrm{s}$.

- Cardiorespiratory fitness will be measured from the 6-min Walk Test and reported as $\mathrm{VO}_{2}$ Peak in $\mathrm{ml} / \mathrm{kg} / \mathrm{min}$ [41]. Heart rate, blood pressure, rate pressure product (systolic arterial pressure $\mathrm{x}$ heart rate) and $\mathrm{VO}_{2}$ Peak will be recorded at rest and during the 6-min Walk Test, by a fully portable compact metabolic system using breath by breath analysis (Metamax 3B, Cortex, Biophysik, Germany) shown to be stable over time and accurate when tested against a gold standard criterion [42]. Test-retest reliability of cardiorespiratory variables measured using the Metamax $3 \mathrm{~B}$ has been demonstrated for people with stroke when completing the 6-min Walk Test (ICCs range 0.76-0.97, error < 4\%) [43].

- Cardiovascular risk will be measured from lipid profile and inflammatory markers. A finger stick will be taken and immediately analysed on a point of care device (Cholestech LDX, Alere ${ }^{\mathrm{mu}}$, Brisbane) [44] for lipid profile: total cholesterol (TC), low density lipoprotein (LDL), high density lipoprotein (HDL), ratio of total cholesterol to HDL (TC/HDL) and triglycerides (TRG). This instrument has been shown to have excellent validity compared to gold standard laboratory analysis [44]. Lipid profile measures for the Cholestech LDX had ICCs ranging from $0.87-0.99$. A blood sample will be taken and analysed for inflammatory markers (high sensitivity CRP) [25].

- Self-efficacy of walking ability will be measured using the Ambulatory Self-Confidence Questionnaire, which has demonstrated reliability and validity in the older population [45]. The 22 items will be reported as a score from 0 to 10 where 0 is no confidence.

- Depression will be measured using the Hospital and Anxiety Depression Scale [46] and reported as a score from 0 to 21 on the depression subscale, where 0 is no depression. It has established validity in people with stroke [47].

- Perception of physical activity will be measured using the Physical Activity Scale for Individuals with Physical Disabilities in the domains of home repair, lawn and garden work, housework, vigorous sport and recreation, moderate sport and recreation, and occupation and transportation and the 13 items reported as a score from 0 to $100 \mathrm{MET}$ hr./day, where 0 is no activity [48]. This survey has demonstrated criterion validity and test-retest reliability [49].

- Participation will be measured using the Impact on Participation and Autonomy Questionnaire [50] in the domains of indoor and outdoor autonomy, family role, social relations, work and education and the 31 items reported as a score from 30 to 155 , where 30 is very little impact. It has demonstrated validity and test-retest reliability (ICC 0.83 to 0.91 ) in people with chronic disorders [51].

- Health-related quality of life will be measured using the EuroQual-5D [52] in the domains of mobility, usual activities, personal care, pain/ discomfort and anxiety/depression as well as a measure of overall health state using a visual analogue scale. The domains will be reported as a utility score between 0 and 1 , and the overall health state reported between 0 and 100 , where 0 is poor health. It has established psychometric properties in people with stroke [53].

Data quality will be maintained by assessors being experienced physiotherapists trained in the collection of outcome measures. Double data entry will be performed separately by two research assistants blind to group allocation to reduce the potential for transcription error. All data will be de-identified to maintain confidentiality during and after the trial. Participants will receive a summary of results at the completion of the trial. 


\section{Sample size}

One hundred twenty eight participants will be recruited. The sample size has been calculated to be able to detect an effect size of 1200 steps/day with $80 \%$ power at a two-tailed significance level of 0.05 . This between-group difference was based on an estimate that participants would take 4000 (SD 2000) steps/day at baseline and that the control intervention would increase this to 5200 step/day and the experimental intervention would increase this to 6400 steps/day, putting them above a sedentary level for older adults [54]. The smallest number of participants to detect a difference in physical activity of 1200 steps/day between two groups estimated from independent samples is 55 participants per group. On the assumption from previous trials with this population that approximately $15 \%$ of the participants may be lost to follow up during the course of the study, we have set a target of 128 participants in total. In order to reach the target sample size, the trial will be carried out across multiple centres, and a researcher will be employed to screen all stroke survivors at each centre.

\section{Statistical analysis}

Primary and secondary outcomes including physical activity, walking ability, cardiorespiratory fitness, cardiovascular risk, self-efficacy, perception of physical activity, participation, and quality of life will be reported as point estimates and measures of variability. An intention-totreat between-group analysis will be performed on all outcome measures. Between-group differences will be analysed using generalized mixed effects models for continuous data, since this analysis does not require casewise deletion due to missing data. Data will be presented as mean difference $(95 \% \mathrm{CI})$. Baseline characteristics judged to be clinically different between groups may be included as covariates.

\section{Discussion}

This study has the potential to reduce disability and burden of care in people with a major health condition that has a high personal and community cost. Heart, stroke and vascular diseases are collectively one of the leading causes of premature death and disability in the developed world. More than $75 \%$ of people with stroke have coronary heart disease and are 2-3 times more likely to be hospitalised with coronary heart disease and heart failure than the rest of the population [55], which contributes to high health costs. Low levels of physical activity are a known risk factor of coronary heart disease. After stroke, people have low levels of physical activity. This research will provide evidence of the effect of the application of a commercially available technology (treadmill training), used in a manner (high-intensity) that will enable stroke survivors to increase their fitness, thereby improving physical activity in the short-term. It is also anticipated that the self-management aspect of the intervention will result in the maintenance of high physical activity in the long-term, thereby improving quality of life and reducing the burden on others. Given the major demographic shift in developed nations involving significant growth in the aged population, this research could make an important evidence-based contribution to the promotion of healthy ageing.

\section{Acknowledgements}

Not applicable.

\section{Funding}

This study has been funded by the National Health and Medical Research Council of Australia. These government independent funding bodies have no role in the design of the study or collection, analysis, and interpretation of data or in writing the manuscript.

\section{Availability of data and materials}

The Creative Commons Public Domain Dedication waiver (https:// creativecommons.org/publicdomain/zero/1.0/) will apply to the data produced from this study.

Trial identifier

Australian New Zealand Clinical Trials Registry (ACTRN12613000744752). National Health and Medical Research Council of Australia (GNT1047426).

\section{Sources and types of support}

This study has been funded by the National Health and Medical Research Council of Australia. This government funding body is independent and has no role in the design of the study or collection, analysis, and interpretation of data or in writing the manuscript.

\section{Trial sponsor}

The University of Queensland, St Lucia, Brisbane, Queensland 4072, AUSTRALIA.

\section{Authors' contributions}

$S B$, LA and SK conceived this study. SB, LA, SK and JP contributed to the design of the study and the procurement of funding. SB, LA and SK drafted the manuscript and all authors have read and approved the final manuscript.

\section{Ethics approval and consent to participate}

This study has been approved by the Metro South Health Service District Human Research Ethics Committee (EC00167) and the University of Queensland Human Research Ethics Committee (2013000639). Informed consent will be gained from each participant face to face by a member of the research team before data collection.

\section{Consent for publication}

Not applicable.

\section{Competing interests}

The authors declare that they have no competing interests.

\section{Publisher's Note}

Springer Nature remains neutral with regard to jurisdictional claims in published maps and institutional affiliations.

\section{Author details}

${ }^{1}$ Discipline of Physiotherapy, School of Health and Rehabilitation Sciences, The University of Queensland, St Lucia, QLD, Australia. ${ }^{2}$ School of Physiotherapy, Faculty of Health Sciences, Australian Catholic University, Banyo, QLD, Australia. ${ }^{3}$ School of Allied Health Sciences, Griffith University, Southport, QLD, Australia. ${ }^{4}$ Discipline of Physiotherapy, Faculty of Health Sciences, The University of Sydney, Sydney, NSW, Australia. 
Received: 8 June 2017 Accepted: 3 January 2018

Published online: 30 January 2018

\section{References}

1. Sen S, Lynch DR Jr, Kaltsas E, Simmons J, Tan WA, Kim J, et al. Association of asymptomatic peripheral arterial disease with vascular events in patients with stroke or transient ischaemic attack. Stroke. 2009:40:3472-7.

2. Hartmann A, Rundek T, Mast H, Paik MC, Boden-Albala B, Mohr JP, et al. Mortality and causes of death after first ischaemic stroke: the northern Manhattan stroke study. Neurology. 2001;57:2000-5.

3. Lee CDE, Folsom A, Blair S. Physical activity and stroke risk: a meta-analysis. Stroke. 2003;34:2475-81.

4. Wendel-Vos GC, Schuit AJ, Feskens EJ, Boshuizen HC, Verschuren WM, Saris WH, et al. Physical activity and stroke. A meta-analysis of observational data. Int J Epidem. 2003;33:787-98.

5. D'Isabella NT, Shkredova DA, Richardson JA, Tang A. Effects of exercise on cardiovascular risk factors following stroke or transient ischemic attack: a systematic review and meta-analysis. Clin Rehabil. 2017; May 1: 269215517709051. doi: https://doi.org/10.1177/0269215517709051.

6. Gordon NF, Gulanick M, Costa F, Fletcher G, Franklin BA, Roth EJ, et al. Physical activity and exercise recommendations for stroke survivors. Circulation. 2004:109:2031-41.

7. Tully MA, Cupples ME, Chan WS, McGlade K, Young IS, et al. Brisk walking, fitness and cardiovascular risk: a RCT in primary care. Prev Med. 2005;41:622-8.

8. Hartman-Maeir A, Soroker N, Ring H, Avni N, Katz N. Activities, participation and satisfaction one-year post stroke. Disabil Rehabil. 2007;29:559-66.

9. Rand D, Eng JJ, Tang PF, Jeng JS, Hung C. How active are people with stroke?: use of accelerometers to assess physical activity. Stroke. 2009;40:163-8.

10. Boysen $\mathrm{G}$, Krarup LH. Benefits of physical activity for stroke survivors. Expert Rev Neurother. 2009:9:147-9.

11. Alzahrani MA, Ada L, Dean CM. Duration of physical activity is normal but frequency is reduced after stroke: an observational study. J Physiother. 2011;57:47-51.

12. Lord SE, McPherson K, McNaughton HK, Rochester L, Weatherall M. Community ambulation after stroke: how important and obtainable is it and what measures appear predictive? Arch Phys Med Rehabil. 2004:85:234-9.

13. Resnick B, Shaughnessy M, Nahm ES, Kobunek S, Sorkin J, Orwig D, et al. Inflated perceptions of physical activity after stroke: pairing self report with physiologic measures. J Phys Act Health. 2008;5:308-18.

14. Shaughnessy M, Resnick BM, Macko RF. Testing a model of post stroke exercise behavior. Rehabil Nurs. 2006;31:15-21.

15. Saunders DH, Sanderson M, Hayes S, Kilrane M, Greig CA, Brazzelli M, et al. Physical fitness training for stroke patients. Cochrane Database Syst Rev. 2016;3:CD003316. https://doi.org/10.1002/14651858.CD003316.pub6.

16. Marsden DL, Dunn A, Callister R, Levi CR, Spratt NJ. Characteristics of exercise training interventions to improve cardiorespiratory fitness after stroke: a systematic review with meta-analysis. Neurorehabil Neural Repair. 2013:27:77588. https://doi.org/10.1177/1545968313496329. Epub 2013 Jul 24.

17. Kuys SS, Brauer SG. Ada L higher-intensity treadmill walking during rehabilitation after stroke is feasible and not detrimental to walking pattern or quality: a pilot randomised trial. Clin Rehabil. 2011;25:316-26.

18. Mead G, Bernhardt J. Physical fitness training after stroke, time to implement what we know: more research is needed. Int J Stroke. 2011;6:506-8.

19. Jones F, Riazi A. Self-efficacy and self-management after stroke: a systematic review. Disabil Rehabil. 2011:33:797-810.

20. Schwarzer R, Lippke S, Luszczynska A. Mechanisms of health behavior change in persons with chronic illness or disability: the health action process approach (HAPA). Rehabil Psychol. 2011;56:161-70.

21. Lippke S, Ziegelmann JP, Schwarzer R. Initiation and maintenance of physical exercise: stage-specific effects of a planning intervention. Res Sports Med. 2004;12:221-40.

22. Schwarzer R, Luszczynska A, Ziegelmann JP, Scholz U, Lippke S. Socialcognitive predictors of physical exercise adherence: three longitudinal studies in rehabilitation. Health Psychol. 2008;27:S54.

23. Locke EA, Latham GP. Building a practically useful theory of goal setting and task motivation. Am Psychol. 2002;57:705-17.

24. Roth EJ. Heart disease in patients with stroke: incidence, impact, and implications for rehabilitation part 1: classification and prevalence. Arch Phys Med Rehabil. 1993;74:752-60
25. Carrado E, Rizzo M, Coppola G, Fattouch K, Novo G. Marturana et al. an update on the role of markers of inflammation in atherosclerosis. J Atheroscl Thromb. 2010;17:1-11.

26. Gialluira F, Lucci R, D'Agostino M, Vitelli A, Maresca L, Mancini M, et al. Two year multicomprehensive secondary prevention program: favourable effects on cardiovascular functional capacity and coronary risk profile after acute myocardial infarction. J Cardiovasc Med. 2009;10:772-80.

27. Milani RV, Lavie CJ, Mehra MR. Reduction in C-reactive protein through cardiac rehabilitation and exercise training. J Am Coll Cardiol. 2004:43:1056-61.

28. Kraus WE, Slentz CA. Exercise training, lipid regulation and insulin action: a tangled web of cause and effect. Obesity. 2009;17:S21-6.

29. Lakka TA, Lakka HM, Rankinen T, Leon AS, Rao DC, Skinner JS, et al. Effect of exercise training on plasma levels of C-reactive protein in healthy adults: the HERITAGE family study. Eur Heart J. 2005;26:2018-25.

30. Ivey FM, Ryan AS, Hafer-Mako CE, Macko RF. Improved cerebral vasomotor reactivity after exercise training in hemiparetic stroke survivors. Stroke. 2011; 42:1994-2000.

31. Beller EM, Gebski V, Keech AC. Randomisation in clinical trials. Med J Aust. 2002;177:565-7.

32. National Stroke Foundation. Clinical guidelines for stroke management. Australia: National Stroke Foundation; 2010.

33. Borg G. Borg's perceived exertion and pan scales. Champaign: Human Kinetics; 1998.

34. American College of Sports Medicine. ACSM's guidelines for exercise testing and prescription. 7th ed. Baltimore: Lippincott Williams \& Wilkins; 2006.

35. Schwarzer R. Self-efficacy in the adoption and maintenance of health behaviors: theoretical approaches and a new model. In Schwarzer, R. (Ed.), Self-efficacy: thought control of action. Washington DC: Hemisphere; 2002. p217-243.

36. Locke EA, Latham GP. A theory of goal setting and task performance. Prentice Hall: Englewood Cliffs; 1990.

37. Mahendran N, Kuys SS, Downie E, Ng P, Brauer SG. Are accelerometers and GPS devices valid, reliable and feasible tools for measurement of community ambulation after stroke? Brain Impair. 2016;17:151-61.

38. Enright PL. The six-minute walk test. Resp Care. 2003;48:783-5.

39. Solway $\mathrm{S}$, et al. A qualitative systematic review of the measurement properties of functional walk tests used in the cardiorespiratory domain. Chest. 2001;119:256-70.

40. Fulk GD, et al. Clinimetric properties of the six-minute walk test in individuals undergoing rehabilitation post stroke. Physiother Theory Prac. 2008;24:195-204.

41. Dale RA, Jensen LH, Krantz MJ. Comparison of two point-of-care lipid analyzers for use in global cardiovascular risk assessments. Ann Pharmacother. 2008;42:633-9.

42. MacFarlane DJ, Wong P. Validity, reliability and stability of the portable cortex Metamax 3B gas analysis system. Eur J Appl Physiol. 2012;112:2539-47.

43. Polese JC, Ada L, Parreira VF, Faria GS, Avelino P, Teixeira-Salmela LF. Test-retest reliability of cardiorespiratory variables measured with the Metamax 3B during the six minute walk test after stroke. Phys Med Rehabil Int. 2015;2:1028

44. Carey M, Markham C, Gaffney P, Boran C, Maher V. Validation of a point of care lipid analyser using a hospital based reference system. Ir J Med Sci. 2006;175:30-5.

45. Asano M, Miller WC, Eng JJ. Development and psychometric properties of the ambulatory self-confidence questionnaire. Gerontol. 2007:53:373-81.

46. Zigmond AS, Snaith RP. The hospital anxiety and depression scale. Acta Psychiatr Scand. 1983:67:361-70.

47. Aben I, Verhey F, Lousberg R, Lodder J, Honig A. Validity of the beck depression inventory, hospital anxiety and depression scale, SCL-90, and hamilton depression rating scale as screening instruments for depression in stroke patients. Psychosomatics. 2002;43:386-93.

48. Washburn RA, Zhu W, McAuley E, Frogley M, Figoni SF. The physical activity scale for individuals with physical disabilities: development and evaluation. Arch Phys Med Rehabil. 2002:83:193-200.

49. van der Ploeg HP, Streppel KR, van $\operatorname{der}$ Beek AJ, van der Woude LH, Vollenbroek-Hutten M, van Mechelen W. The physical activity scale for individuals with physical disabilities: test-retest reliability and comparison with an accelerometer. J Phys Act Health. 2007:4:96-100

50. Cardol M, de Haan RJ, van den Bos GA, de Jong BA, de Groot IJ. The development of a handicap assessment questionnaire: the impact on participation and autonomy. Clin Rehabil. 1999;13:411-9. 
51. Cardol M, de Haan RJ, de Jong BA, et al. Psychometric properties of the impact on participation and autonomy questionnaire. Arch Phys Med Rehabil. 2001;82:210-6.

52. EuroQol Group. EuroQol-a new facility for the measurement of a healthrelated quality of life. Health Policy. 1990;16:199-208.

53. Golicki G, Niewada M, Buczek J, Karlinska A, Kobayashi A, Janssen MF, et al. Validity of EQ-5D-5L in stroke. Qual Life Res. 2015;24:845-50.

54. Tudor-Locke C, Jones R, Myers AM, Paterson DH, Ecclestone NA.

Contribution of structured exercise class participation and informal walking for exercise to daily physical activity in community dwelling older adults. Res Quart Ex Sport. 2002;73:350-6.

55. Australian Institute of Health and Welfare. Heart, Stroke and Vascular Diseases: Australian Facts, 2004

Submit your next manuscript to BioMed Central and we will help you at every step:

- We accept pre-submission inquiries

- Our selector tool helps you to find the most relevant journal

- We provide round the clock customer support

- Convenient online submission

- Thorough peer review

- Inclusion in PubMed and all major indexing services

- Maximum visibility for your research

Submit your manuscript at www.biomedcentral.com/submit
Biomed Central 